\title{
271. Autologe Spongiosa bei Osteomyelitis mit Knochen-, Weichteil- und Hautdefekt
}

\author{
C. BURRI* und H. HenkemeYer-Ulm
}

\author{
Autogenous Cancellous Bone in Osteomyelitis with Defects \\ of Bone, Soft Tissue and Skin
}

Summary. Surgeons are still confronted with the grave problem of the "unhappy triad" of traumatology, i.e. infection, osseous defect and instability. To us the stabilisation of fragments by means of internal or external fixation, the radical saucerization and packing of the cavity with autogenous cancellous bone with preceding irrigation drainage seems to be the procedure best suitable. 25 patients with infected defects of bone, soft tissue and skin were treated accordingly and followed up 1 to 6 years later: In all cases the graft had been integrated and the skin- and soft tissue defects had healed. In 23 cases osteomyelitis had not reoccured, weight bearing stability had been achieved in all 25 cases.

The described procedure had either maintained or even improved articular function. Additionally the active intervention allowes correction of axis and length.

It is demonstrated by radiological, scintigraphical and histological examinations that the integration of the cancellous bone transplant begins immediately after transplantation and is advanced within three months to such a point that weight bearing becomes possible.

Zusammenfassung. Die „unhappy triad“ der Knochenchirurgie, Infekt, Defekt und Instabilität stellt uns auch heute noch vor schwer zu lösende Probleme. Das Vorgehen der Wahl scheint uns die Stabilisierung der Fragmente mittels einer internen (Osteosynthese) oder externen (äußere Spanner) Fixation, die radikale Ausräumung des Herdes, die vorübergehende Spüldrainage und schließlich das Auffüllen des Defektes mit autologer Spongiosa zu sein. Wir haben 25 Patienten mit infiziertem Knochen-, Weichteil- und Hautdefekt nach diesen Prinzipien behandelt und dieses Kollektiv 1-6 Jahre später nachkontrolliert: In sämtlichen Fällen kam es zum knöchernen Einbau des Transplantates und Abheilung des Hautund Weichteildefektes. 23 Patienten sind 1-6 Jahre nach der Behandlung vom Infekt her rezidivfrei geblieben, bei sämtlichen Patienten konnte Belastungsstabilität erreicht werden.

Durch das beschriebene Vorgehen konnte in allen Fällen die Gelenkfunktion erhalten oder verbessert werden. Das aktive Eingreifen gestattet zudem Achsenund Längenkorrekturen.

Radiologische, szintigraphische und histologische Untersuchungen zeigen, daß der Einbau des spongiösen Transplantates unmittelbar nach der Verpflanzung einsetzt und nach 3 Monaten soweit fortgeschritten ist, daß die Belastungsstabilität erreicht wird.

Leiter: Herr Burri, ich darf noch einmal feststellen: Sie legen in eine infizierte Defektpseudarthrose autologe Spongiosa offen ein und fixieren sie durch eine Osteosynthese?

Burri-Ulm: Die Reihenfolge des Vorgehens ist etwas anders. Zuerst Fixation mit Ausräumung und Spüldrainage. Dann nach vierwöchiger Spüldrainage, wenn das Lager mechanisch gereinigt ist, wird die Spongiosa eingebracht.

Leiter: Es scheint mir wichtig, das festzustellen. Denn die Väter der Transplantationschirurgie würden hier sehr erhebliche Bedenken haben. Aber ich gratuliere Ihnen zu den schönen Erfolgen. 